\title{
SNAP-25 is a promising novel cerebrospinal fluid biomarker for synapse degeneration in Alzheimer's disease
}

\author{
Ann Brinkmalm 1*, Gunnar Brinkmalm¹, William G Honer ${ }^{2}$, Lutz Frölich ${ }^{3}$, Lucrezia Hausner ${ }^{3}$, Lennart Minthon ${ }^{4,5}$, \\ Oskar Hansson ${ }^{4,5}$, Anders Wallin ${ }^{1}$, Henrik Zetterberg ${ }^{1,6}$, Kaj Blennow $^{1}$ and Annika Öhrfelt ${ }^{1}$
}

\begin{abstract}
Background: Synaptic degeneration is an early pathogenic event in Alzheimer's disease, associated with cognitive impairment and disease progression. Cerebrospinal fluid biomarkers reflecting synaptic integrity would be highly valuable tools to monitor synaptic degeneration directly in patients. We previously showed that synaptic proteins such as synaptotagmin and synaptosomal-associated protein 25 (SNAP-25) could be detected in pooled samples of cerebrospinal fluid, however these assays were not sensitive enough for individual samples.
\end{abstract}

Results: We report a new strategy to study synaptic pathology by using affinity purification and mass spectrometry to measure the levels of the presynaptic protein SNAP-25 in cerebrospinal fluid. By applying this novel affinity mass spectrometry strategy on three separate cohorts of patients, the value of SNAP-25 as a cerebrospinal fluid biomarker for synaptic integrity in Alzheimer's disease was assessed for the first time. We found significantly higher levels of cerebrospinal fluid SNAP-25 fragments in Alzheimer's disease, even in the very early stages, in three separate cohorts. Cerebrospinal fluid SNAP-25 differentiated Alzheimer's disease from controls with area under the curve of $0.901(P<0.0001)$.

Conclusions: We developed a sensitive method to analyze SNAP-25 levels in individual CSF samples that to our knowledge was not possible previously. Our results support the notion that synaptic biomarkers may be important tools for early diagnosis, assessment of disease progression, and to monitor drug effects in treatment trials.

Keywords: Alzheimer's disease, Biomarker, Cerebrospinal fluid, SNAP-25, SNARE proteins, Mass spectrometry, Immunopurification, Selected reaction monitoring

\section{Background}

Animal models of the early phases of Alzheimer's disease have directly demonstrated loss of presynaptic proteins and synaptic dysfunction [1,2]. In patients, however, assays for presynaptic proteins are indirect or rely on post-mortem findings. In the early stages of disease they have provided inconsistent results reporting elevated, unchanged, and lower protein amounts [3-12]. Biomarker studies of amyloid $\beta 1-42$ (A $\beta 1-42)$, total tau (T-tau) and tau phosphorylated at threonine $181\left(\mathrm{P}-\mathrm{tau}_{181}\right)$ in cerebrospinal fluid (CSF) have contributed to understanding the

\footnotetext{
* Correspondence: ann.brinkmalm@neuro.gu.se

'Institute of Neuroscience and Physiology, Department of Psychiatry and Neurochemistry, Sahlgrenska Academy at the University of Gothenburg, S-431 80 Mölndal, Sweden

Full list of author information is available at the end of the article
}

sequence of clinically relevant molecular events contributing to cognitive impairment [13]. In the central nervous system synaptosomal-associated protein 25 (SNAP-25) is an important marker of functional synapses, being one essential component of the soluble N-ethylmaleimidesensitive factor attachment protein receptors (SNARE) complex. These proteins mediate synaptic communication by initiating fusion of synaptic vesicles [14].

The notions that synaptic loss occurs early in Alzheimer's disease, and that synaptic proteins at active synapses could be biomarkers indicating the degree of synaptic degeneration have prompted interest in detecting relevant synaptic proteins in human biological fluid samples. Analysis of synaptic proteins in CSF is complicated by the presence of only trace amounts, and the membrane-bound nature of 
many of these proteins [15]. Several research groups, including our own, have detected synaptic proteins in CSF [16-21]. However, these studies were performed on relatively large quantities of pooled CSF from multiple patients $[16,17,21]$. Moreover, the target proteins had to be selectively purified and concentrated in several steps and the quantitative aspects of the techniques may have been sub-optimal [20].

Here, we have developed an assay where the concentration of the presynaptic protein SNAP-25 could be reproducibly measured in CSF samples from individual patients. We hypothesized that soluble forms of brain SNAP-25 were the most likely to resemble SNAP-25 in CSF. Since SNAP-25 is abundant in brain tissue we used biochemically fractionated human brain homogenate (soluble, membrane-bound, and membrane-raft associated protein fractions) to design a strategy for quantification of SNAP-25 in CSF by combining selective purification with immunoprecipitation, digestion with trypsin, and mass spectrometry analysis. We found significantly higher levels of SNAP-25 in CSF in Alzheimer's disease in three separate cohorts, including in the very early stage of the disease.

\section{Results}

Characterization and quantification of SNAP-25 in human brain tissue

We used the monoclonal antibodies to affinity purify SNAP-25 from biochemically fractionated human brain homogenate. Using the SP12 antibody and quantification with selected reaction monitoring mass spectrometry (SRM-MS) we compared the SNAP-25 levels in brain homogenate fractions from Alzheimer's disease patients $(\mathrm{N}=15)$ and age-matched controls $(\mathrm{N}=15)$ (Additional file 1: Table S1). We found that the levels of SNAP-25 were significantly lower in the Alzheimer's disease group for the membrane-bound and the membrane-raft associated fractions (Figure 1A-B). In contrast, the levels of SNAP-25 in the soluble protein fractions were very low or undetectable (data not shown). However, when affinity purifying with SMI81 instead of SP12, SNAP-25 could be quantified in all soluble fractions (Figure 1C). Nevertheless, no significant differences between the levels of soluble SNAP-25 in Alzheimer's disease and control brain homogenate samples were observed.

To investigate if the dissimilar levels of SP12- and SMI81-immunoreactive SNAP-25 in the soluble fractions could be due to a truncation or other post-translational modifications we analyzed a subset of the samples with LC-MS/MS. In the majority of the soluble fractions, only tryptic peptides originating from the N-terminal part of the SNAP-25 protein were detected (Figure 2), indicating that the soluble SNAP-25 were C-terminally truncated. However, in all the membrane-bound and membrane-raft associated fractions the entire SNAP-25 protein was detected (regardless of antibody) (Additional file 1: Figure S1). Moreover, in all fractions, including the soluble, SNAP-25 was N-terminally modified by methionine excision and acetylation.

To characterize the soluble SNAP-25 forms we used a top-down LC-MS/MS approach. Using undigested proteins from the SMI81 affinity purified soluble fraction, we successfully identified eight truncated forms of soluble SNAP-25, all N-terminally modified by methionine excision and acetylation (Additional file 1: Figure S2, Table S2). Building on the findings of the soluble truncated SNAP-25 peptides we changed our approach to target the furthermost N-terminal tryptic peptides of SNAP-25 (Figure 2B) and perform the MS-based quantification with high resolution selected ion monitoring (HR-SIM-MS) on a Quadrupole-Orbitrap Mass Spectrometer (Q Exactive) [22]. The reproducibility of the novel method was measured and the CV of the SNAP-25 levels was found to be less than 10\% (Additional file 1: Table S3).

\section{Evaluation of SNAP-25 as a synaptic marker in CSF samples \\ Demographic results}

Table 1 shows the demographic characteristics of the groups. The German cohort was composed of nine patients
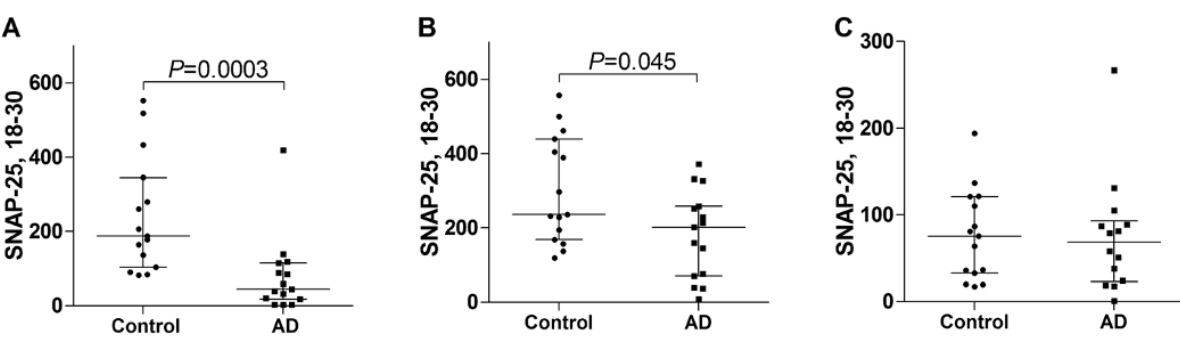

Figure 1 Targeted SRM-MS analyses of SNAP-25 in human brain. Individual values for the SRM-MS measured ratios (endogenous peptide/ labeled peptide standard) of immunoprecipitated [antibodies SP12 (A-B) and SMI81 (C) SNAP-25, in biochemically fractionated membrane-bound (A) and membrane-raft associated (B) and soluble (C), extract of superior parietal gyrus from controls $(\mathrm{N}=15)$ and patients with Alzheimer's disease $(N=15)$. The lower, upper and middle lines of the error bars correspond to the 25th and 75th percentiles and medians, respectively. SMI81 recognizes the extreme N-terminus of SNAP-25, especially when it's N-terminal acetylated. The exact epitope of SP12 is unknown. 


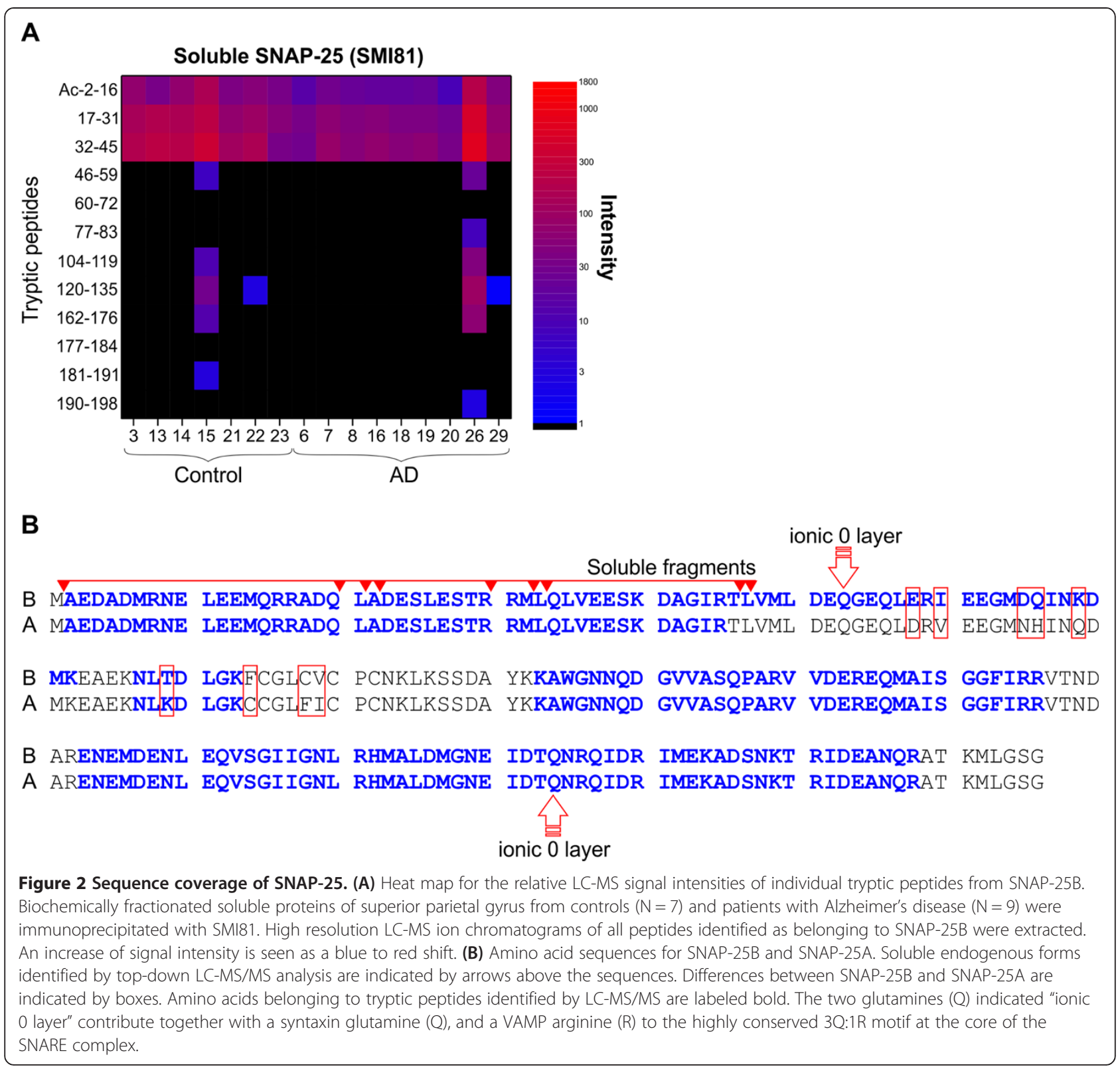

with Alzheimer's disease (three men and six women, 62-83 years), seven subjects with prodromal Alzheimer's disease (four men and three women, 57-77 years), and nine nondemented controls (two men and seven women, 60-83 years). The first replication set (Swedish cohort I) was composed of 10 patients with Alzheimer's disease (three men and seven women, 59-84 years), and six non-demented controls (one man and five women, 47-64 years). The second replication set (Swedish cohort II) was composed of 17 patients with Alzheimer's disease (five men and 12 women, 61-76 years), and 17 healthy controls (nine men and eight women, 63-70 years). The patients and controls in the German and Swedish cohort II were age-matched, while the patients with Alzheimer's disease were significantly older than the controls in the Swedish cohort I. In all three cohorts, patients with Alzheimer's disease had a significantly lower MMSE score compared with the controls.

\section{Levels of SNAP-25 in CSF}

The CSF levels of all three investigated tryptic peptides of SNAP-25 were significantly higher in patients with prodromal Alzheimer's disease and overt Alzheimer's disease compared with non-demented controls (Figure 3A-C). Moreover, two of the SNAP-25 peptides (amino acids 17-31 and 32-40) were significantly higher in Alzheimer's disease compared with prodromal Alzheimer's disease (Figure 3B-C). Consistently, the CSF levels of all tryptic peptides of SNAP-25 were significantly higher in patients 
Table 1 Demographic data and biomarker CSF levels for the diagnostic groups ${ }^{a}$

\begin{tabular}{|c|c|c|c|}
\hline German cohort & Control & Prodromal Alzheimer's disesase & Alzheimer's disease \\
\hline Number (Men/Women) & $9(2 / 7)$ & $7(4 / 3)$ & $9(3 / 6)$ \\
\hline Age (years) & $70(68-74)$ & $72(69-73)$ & $68(68-79)$ \\
\hline MMSE & $27(25-29)$ & $28(27-28)$ & $22(21-23) P=0.02^{b}, P=0.001^{c}$ \\
\hline$A \beta 1-42(n g / L)$ & $1065(797-1201)$ & $541(521-753) P=0.005^{b}$ & $524(424-695) P=0.0005^{b}$ \\
\hline T-tau (ng/L) & $165(135-208)$ & $403(353-513) P=0.0002^{b}$ & $779(683-864) P=0.00004^{b}, P=0.002^{c}$ \\
\hline$P-\operatorname{tau}_{181}(\mathrm{ng} / \mathrm{L})$ & $45(38-50)$ & $81(76-95) P=0.0002^{b}$ & $130(108-161) P=0.00004^{b}, P=0.005^{c}$ \\
\hline Swedish cohort I & Control & Prodromal Alzheimer's disesase & Alzheimer's disease \\
\hline Number (Men/Women) & $6(1 / 5)$ & & $10(3 / 7)$ \\
\hline Age (years) & $54(48-63)$ & & $77(73-82) P=0.001^{\mathrm{b}}$ \\
\hline MMSE & $27(27-28)$ & & $24(22-25) P=0.0003^{b}$ \\
\hline$A \beta 1-42(n g / L)$ & $915(860-1040)$ & & $470(355-560) P=0.0003^{b}$ \\
\hline T-tau (ng/L) & $290(230-300)$ & & $690(590-1100) P=0.0002^{\mathrm{b}}$ \\
\hline P-tau ${ }_{181}(\mathrm{ng} / \mathrm{L})$ & $56(40-60)$ & & $92(84-132) P=0.0003^{b}$ \\
\hline Swedish cohort II & Control & Prodromal Alzheimer's disesase & Alzheimer's disease \\
\hline Number (Men/Women) & $17(9 / 8)$ & & $17(5 / 12)$ \\
\hline Age (years) & $66(64-68)$ & & $68(66-70)$ \\
\hline MMSE & $30(29-30)$ & & $25(24-27) P<0.0001^{b}$ \\
\hline$A \beta 1-42$ (ng/L) & $640(530-870)$ & & $320(210-440) P=0.0001^{b}$ \\
\hline T-tau (ng/L) & $250(180-320)$ & & $560(360-1020) P=0.0006^{\mathrm{b}}$ \\
\hline P-tau 181 (ng/L) & $46(34-58)$ & & $93(54-127) P=0.001^{b}$ \\
\hline
\end{tabular}

Abbreviations: Aß1-42 (amyloid $\beta$ 1-42), CSF (cerebrospinal fluid), MMSE (mini-mental state examination), T-tau (total tau), P-tau 181 (tau phosphorylated at threonine 181).

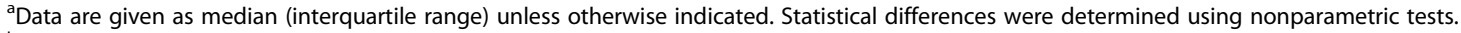

${ }^{\mathrm{b}}$ Compared with controls.

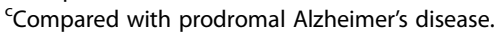

with Alzheimer's disease compared with non-demented controls in the replication set (Figure 3D-F). In the second replication set, the CSF levels of the tryptic peptide of SNAP-25 (32-40) were significantly higher in patients with Alzheimer's disease compared with healthy controls (Figure 3I).

There were no cohort effects on the CSF levels of novel SNAP-25 biomarkers, P-tau 181 or A $31-42$ (data not shown), allowing statistical analyses of the entire group of participant samples from Alzheimer's disease $(\mathrm{N}=36)$ and controls $(\mathrm{N}=32)$ (Figure $3 \mathrm{~J}-\mathrm{L})$. The tryptic peptide assays of SNAP-25 (32-40, 17-31, and Ac-2-16) and CSF biomarkers (A $\beta 1-42$, T-tau and $\mathrm{P}$-tau $\left.\mathrm{ta1}_{181}\right)$ could each differentiate Alzheimer's disease $(\mathrm{N}=36)$ from controls $(\mathrm{N}=32)$, with area under the curve of $0.901(0.828$ 0.974) $(P<0.0001), 0.808(0.703-0.913)(P<0.0001), 0.772$ (0.659-0.885) $(P<0.001), 0.881(0.802-0.960)(P<0.0001)$, $0.933(0.873-0.994)(P<0.0001)$, and $0.916(0.844-0.987)$ $(P<0.0001)$, respectively (Figure 4$)$.

No statistically significant correlations between age and levels of the tryptic peptides of SNAP-25 (Ac-2-16, 17-31, and 32-40) were observed in either the control group $(\mathrm{N}=33)$ or the Alzheimer's disease group $(\mathrm{N}=36)$ (Table 2). The MMSE score, indicating the severity of cognitive impairment, correlated significantly with SNAP-25 (Ac-2-16) in the Alzheimer's disease $(\mathrm{N}=36)$ group (Table 2). All tryptic peptides of SNAP-25 (Ac-2-16, 17-31, and 32-40) correlated with the levels of T-tau and P-tau ${ }_{181}$ in both the control group $(\mathrm{N}=33)$ and in patients with Alzheimer's disease $(\mathrm{N}=36)$ (Table 2). The tryptic peptides of SNAP-25 (Ac-2-16 and 17-31) correlated with A $\beta 1-42$ in the control group, but not in patients with Alzheimer's disease (Table 2).

\section{Discussion}

We report a new strategy to study synaptic pathology by using affinity purification and quantitative mass spectrometry to measure levels of the presynaptic SNAP-25 in CSF samples from individual patients. This is the first study demonstrating that SNAP-25 might be a useful CSF biomarker in differential diagnosis of patients with Alzheimer's disease/prodromal Alzheimer's disease from controls and also in discriminating Alzheimer's disease from prodromal Alzheimer's disease.

The novel CSF SNAP-25 assay was developed using biochemically fractionated human brain homogenate with high concentration of synaptic proteins. We found that the levels of SNAP-25 were significantly lower in 


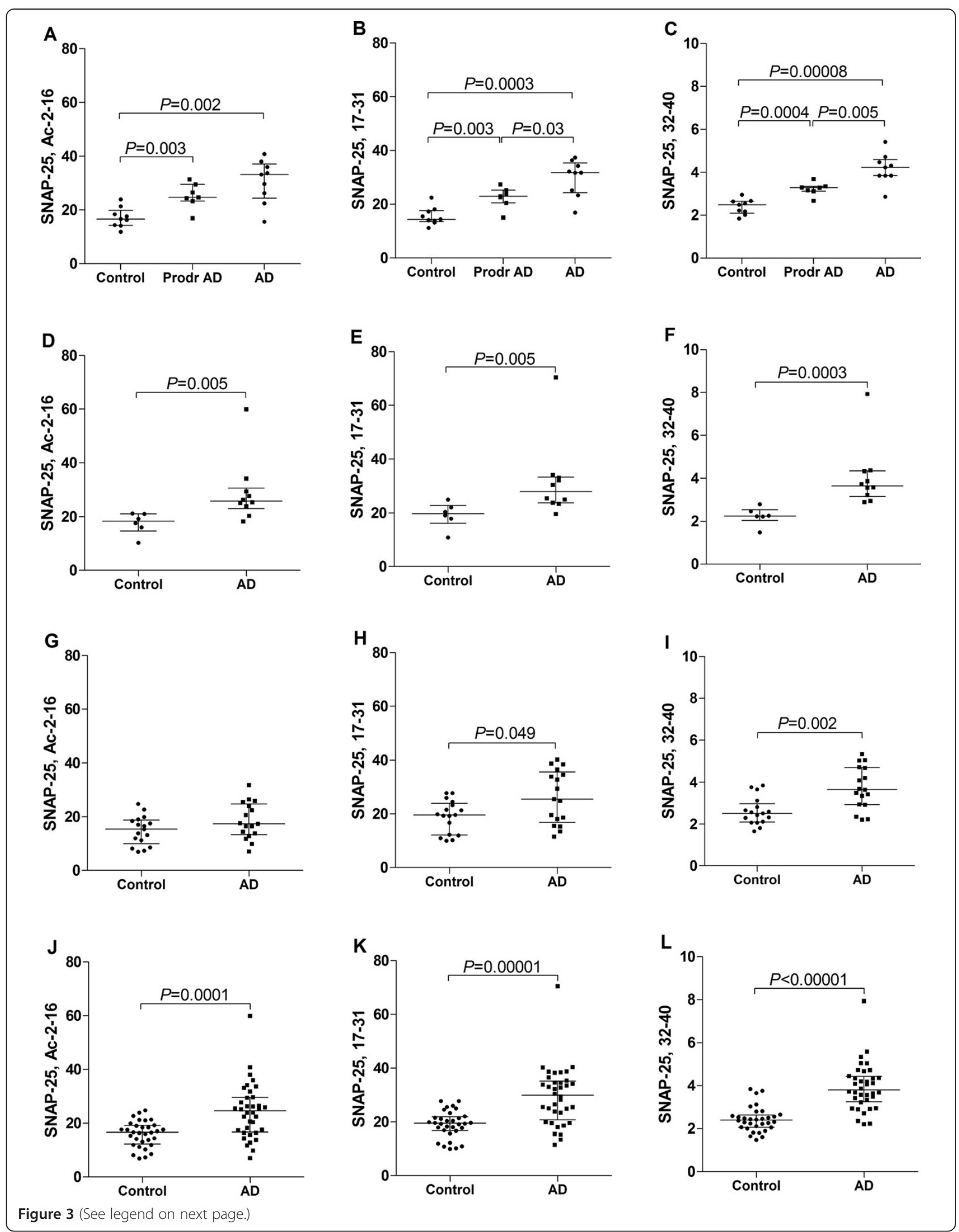



peptide/labeled peptide standard] multiplied by 10,000) of immunoprecipitated (SMI81 antibody) SNAP-25 in CSF samples within German cohort (A-C), Swedish cohort I (D-F), Swedish cohort II (G-I), and the entire group of Alzheimer's disease $(\mathrm{N}=36)$ and controls $(\mathrm{N}=32)(\mathbf{J}-\mathbf{L})$. The nine panels depict the measured levels of three N-terminal tryptic peptides of SNAP-25, Ac-2-16 (A, D, G, J), 17-31 (B, E, H, K), and 32-40 (C, F, I, L).

the Alzheimer's disease group for the membrane-bound and the membrane-raft associated fractions. These results are consistent with previous studies of SNAP-25 in Alzheimer's disease using different immunoassays $[5,6,8,23,24]$ and demonstrated that the method was sufficiently sensitive to detect pathological changes in brain tissue samples from individual patients.

The dissimilar levels of SP12- and SMI81-immunoreactive SNAP-25 in the soluble fractions made us hypothesize that soluble SNAP- 25 may be truncated or modified compared to membrane associated SNAP-25. We found that only tryptic peptides originating from the $\mathrm{N}$-terminal part of the soluble SNAP-25 protein were detected. Moreover, in all fractions, including the soluble, SNAP-25 was $\mathrm{N}$-terminally modified by methionine excision and acetylation, consistent with a previous study [25]. The existence of a soluble N-terminal peptide fragment of SNAP-25 is consistent with the observations that immunoprecipitation with a monoclonal antibody directed towards the N-terminal of SNAP-25 increases the yield.

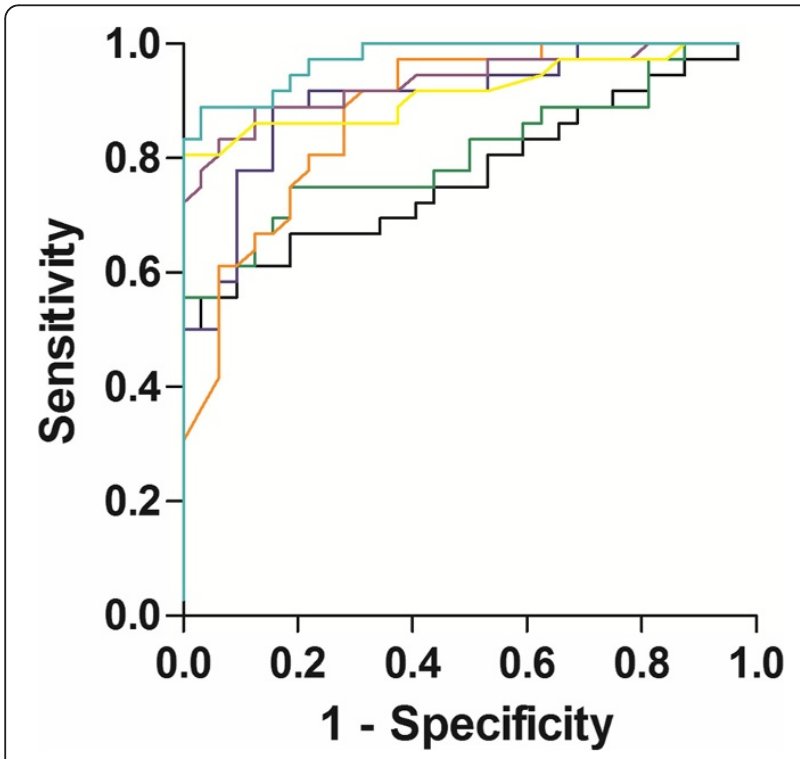

Figure 4 ROC curve analysis of SNAP-25 in human CSF. ROC curve analysis for SNAP-25 32-40 (turquoise), SNAP-25 17-31 (green), SNAP-25 Ac-2-16 (black) Aß1-42 (orange), T-tau (purple) and P-tau 181 (yellow) in CSF for differentiation of Alzheimer's disease patients from controls in the entire subject material. The area under the curve (95\% confidence interval) was $0.901(0.828-0.974)(P<0.0001)$, 0.808 (0.703-0.913) $(P<0.0001), 0.772(0.659-0.885)(P<0.001), 0.881$ (0.802-0.960) $(P<0.0001), 0.933(0.873-0.994)(P<0.0001)$, and 0.916 $(0.844-0.987)(P<0.0001)$, respectively.
Truncated soluble forms of SNAP-25 had never been reported before and we characterized the various forms with a top-down tandem MS approach. Undigested proteins from the SMI81 affinity purified soluble fraction were analyzed directly on LC-MS/MS. We successfully identified eight truncated forms of soluble SNAP-25, all $\mathrm{N}$-terminally modified by methionine excision and acetylation. An interesting finding was that the potential cleavage site for the creation of the longest truncated form of SNAP-25 (Ac-2-47) is located very close to the ionic zero layer at the center of the SNARE complex [26,27] (Figure 2B). However, the longest soluble SNAP-25 contains the $\mathrm{N}$-terminal amino acids $2-47$ of the protein while the two isoforms SNAP-25A and SNAP-25B differs in amino acids 58, 60, 65, 69, 79, 84, and 88-89. Hence, soluble SNAP-25 no longer contains information regarding its original isoform (Figure 2B).

We found that the truncated forms of SNAP-25 were present in CSF, and the level of the tryptic peptide of SNAP-25 (32-40) was consistently and significantly higher in patients with Alzheimer's disease compared with controls in the three independent cohorts. The tryptic peptide assays of SNAP-25 (32-40, 17-31, and Ac-2-16) could each differentiate Alzheimer's disease from controls. However, the tryptic peptide assay of SNAP-25 (32-40) provided a slightly better differentiation of patients with Alzheimer's disease from controls compared with the tryptic assays of SNAP-25 (Ac-2-16 and 17-31). The CSF levels of two of the tryptic peptides of SNAP-25 (Ac-2-16 and 17-31) were significantly higher in patients with Alzheimer's disease compared with controls in two of the three examined clinical cohorts. Summarizing, these findings suggest SNAP-25 (32-40) to provide the best differential diagnostic biomarker of Alzheimer's disease and showed differentiation of patients with Alzheimer's disease from controls in a similar magnitude as the CSF biomarkers (A $\beta 1-42$, T-tau and P-tau 181 ).

In the early stages of disease, synaptic markers in previous studies provided inconsistent results reporting elevated, unchanged, and lower protein amounts [3-12]. In the present study, CSF SNAP-25 peptides were already increased in prodromal Alzheimer's disease compared with controls, supporting the notion that this synaptic marker might provide an early marker for Alzheimer's disease. Two of the SNAP-25 peptides (17-31 and 32-40) could also be used to differentiate prodromal Alzheimer's disease and overt Alzheimer's disease. A limitation of this study is the small sample size for patients with mild 
Table 2 Correlation between age, MMSE and biomarker levels in the entire CSF material ${ }^{\mathrm{a}}$

\begin{tabular}{|c|c|c|c|}
\hline & SNAP-25, Ac-2-16 & SNAP-25, 17-31 & SNAP-25, 32-40 \\
\hline \multicolumn{4}{|l|}{ Cont $(N=33)$} \\
\hline Age & N.S. & N.S. & N.S. \\
\hline MMSE & N.S. & N.S. & N.S. \\
\hline$A \beta 1-42$ & rho $=0.560, P=0.001$ & rho $=0.468, P=0.007$ & N.S. \\
\hline T-tau & rho $=0.564, P=0.001$ & rho $=0.563, P=0.001$ & rho $=0.656, P=0.0001$ \\
\hline P-tau 181 & rho $=0.735, P<0.0001$ & rho $=0.715, P<0.0001$ & rho $=0.659, P<0.0001$ \\
\hline SNAP-25, AC-2-16 & - & rho $=0.859, P<0.0001$ & rho $=0.718, P<0.0001$ \\
\hline SNAP-25, 17-31 & - & - & rho $=0.799, P<0.0001$ \\
\hline \multicolumn{4}{|l|}{$\mathrm{AD}(\mathrm{N}=36)$} \\
\hline Age & N.S. & N.S. & N.S. \\
\hline MMSE & rho $=-0.509, P=0.002$ & N.S. & N.S. \\
\hline$A \beta 1-42$ & N.S. & N.S. & N.S. \\
\hline T-tau & rho $=0.557, P=0.0004$ & rho $=0.732, P<0.0001$ & rho $=0.824, P<0.0001$ \\
\hline P-tau 181 & rho $=0.717, P<0.0001$ & rho $=0.808, P<0.0001$ & $\mathrm{rho}=0.835, P<0.0001$ \\
\hline SNAP-25, AC-2-16 & - & rho $=0.836, P<0.0001$ & rho $=0.720, P<0.0001$ \\
\hline SNAP-25, 17-31 & - & - & rho $=0.900, P<0.0001$ \\
\hline
\end{tabular}

Abbreviations: A 1 1-42 (amyloid $\beta$ 1-42), AD (Alzheimer's disease), CSF (cerebrospinal fluid), MMSE (mini-mental state examination), T-tau (total tau), P-tau 181 (tau phosphorylated at threonine 181).

${ }^{a}$ Correlations presented by the Spearman's rank correlation coefficient (rho). Non significant (N.S.) (P-value $\left.>0.05\right)$ correlations were not reported

cognitive impairment, therefore the value of SNAP-25 as an early biomarker remains to be established.

The patients and controls in two of the cohorts were age-matched, while the patients with Alzheimer's disease were significantly older than the controls in the Swedish cohort I. However, no statistically significant correlations between age and levels of the tryptic peptides of SNAP-25 (Ac-2-16, 17-31, and 32-40) in either the control group or the Alzheimer's disease group were observed, suggesting that the detected SNAP-25 fragments not are influenced by age.

To date there is no CSF biomarker available that makes it possible to follow the progression of cognitive decline. Previous studies suggest that synaptic loss correlates with the clinical manifestations of Alzheimer's disease, while there is no relation between the number of accumulated parenchymal amyloid plaques and synaptic pathology $[3,4]$. In the present study, there was a negative correlation between SNAP-25 (Ac-2-16) and the MMSE, indicating patients suffering from more severe cognitive decline had higher levels of SNAP-25 (Ac-2-16), which implies that the novel biomarker might be useful to follow progression of cognitive decline. Interestingly, previous studies have shown evidence that SNAP-25 single nucleotide polymorphisms are associated with cognitive decline [28,29].

The CSF level of T-tau generally reflects the intensity of axonal and neuronal degeneration occurring in brain, while P-tau ${ }_{181}$ serves as a more specific marker for Alzheimer's disease [30] CSF T-tau, P-tau 181 and A $\beta 1-42$ are stable over time making these Alzheimer's biomarkers feasible for monitoring biochemical effects in clinical trials [31]. The finding that all investigated SNAP-25 peptides correlated well with T-tau and P-tau 181 , suggests that SNAP-25 might be a useful as a surrogate biomarker in future clinical treatment studies with tau modifying drugs [32].

\section{Conclusions}

In summary, we have developed an assay allowing reproducible measurement of the level of the presynaptic protein SNAP-25 in CSF samples from individual patients. We demonstrate significantly higher levels of SNAP-25 in CSF samples from patients with prodromal Alzheimer's disease and Alzheimer's disease compared with controls. Our results show that SNAP-25 is a promising novel CSF biomarker for synapse degeneration in Alzheimer's disease. This finding could be important for earlier diagnosis, assessment of progression of disease and to monitor drug effects in treatment trials in neurodegenerative diseases. We also report the identification of previously unknown, truncated soluble forms of SNAP-25 that could be employed to study the dynamics of SNARE protein processing and recycling.

\section{Methods}

\section{Human brain tissue samples}

The study included autopsy-confirmed patients with Alzheimer's disease $(\mathrm{N}=15)$ and age-matched controls $(\mathrm{N}=15)$. Brain tissues from the region superior parietal gyrus were analyzed. All brain tissues were obtained 
from the Netherlands Brain Bank. Braak and Braak criteria, which are based on the distribution of neurofibrillary tangles, were used to categorize the stage of Alzheimer's disease [33]. All Alzheimer's disease patients fulfilled Braak stages 5 or 6 , while the controls fulfilled Braak stages 0 or 1 . Additional file 1: Table S1 shows the clinical and demographic characteristics of the groups.

\section{CSF samples}

The exploratory phase of the investigation was performed on pooled decoded CSF samples supplied by the Clinical Neurochemistry Laboratory, Sahlgrenska University Hospital Sweden, from patients who underwent lumbar puncture to exclude infectious disorders of the central nervous system.

\section{The German cohort}

CSF samples were obtained at the Interdisciplinary Memory Clinic of the Department of Geriatric Psychiatry of the Clinic of Psychiatry at the Central Institute of Mental Health, Mannheim from subjects with Alzheimer's disease $(\mathrm{N}=9)$, prodromal Alzheimer's disease $(\mathrm{N}=7)$ and nondemented controls $(\mathrm{N}=9)$ (Table 1). Alzheimer's disease was diagnosed according to the NINCDS-ADRDA criteria, with all Alzheimer's disease patients fulfilling the criteria for probable Alzheimer's disease [34]. Mild cognitive impairment due to Alzheimer's disease was diagnosed according the new research criteria of Albert et al in 2011 [35]. Mild cognitive impairment was considered due to prodromal Alzheimer's disease if additionally, biomarkers of molecular neuropathology of Alzheimer's disease in CSF were measured positively for Alzheimer's disease (CSF biomarkers A $\beta 1-42 \leq 450$ ng/L; T-tau $\geq 450$ ng/L; P-tau $181 \geq 61 \mathrm{ng} / \mathrm{L}$ ) or if there was hippocampal volume reduction or medial temporal atrophy assessed by visual rating (Schelten's scale $>2$ ) measured by an experienced neuroradiologist. Non-demented controls had various psychiatric diagnoses, (including geriatric depression, and schizophrenia), Lumbar puncture in these patients was carried out for clinical indications, such as excluding organic brain disorder. All were found normal on cognitive screening tests, all routine CSF analyses were within normal limits and none of the CSF biomarkers were positive for Alzheimer's disease.

\section{The Swedish cohort I}

CSF samples were obtained at the Memory Clinic at Skåne University Hospital in Malmö from subjects with Alzheimer's disease $(\mathrm{N}=10)$ and non-demented controls $(\mathrm{N}=6)$ (Table 1). Subjects diagnosed with Alzheimer's disease met the DSM-III-R criteria for dementia [36] and the criteria for probable Alzheimer's disease, as defined by NINCDS-ADRDA [34]. The non-demented cases exhibited cognitive complaints, but did not fulfil the criteria for dementia. To rule out preclinical Alzheimer's disease in the latter group we only included cases with normal CSF A $31-42>550$ ng/L and T-tau <400 ng/L levels.

\section{The Swedish cohort II}

CSF samples from subjects with Alzheimer's disease $(\mathrm{N}=17)$ and healthy controls $(\mathrm{N}=17)$ were obtained from the Gothenburg mild cognitive impairment study for which the diagnostic procedure was described in detail previously [37] (Table 1). The diagnosis of dementia was based on the DSM-III-R criteria [36] together with the criteria of NINCDS-ADRDA [34] and ICD-10 [38] with regard to Alzheimer's disease. Controls were not included if they had subjective or objective signs of a cognitive disorder.

\section{CSF collection}

All CSF samples were obtained by lumbar puncture though the L3/L4 interspace. The CSF samples were centrifuged at 2,000 $\mathrm{g}$ for $10 \mathrm{~min}$ at room temperature to remove cells and debris, and stored in aliquots at $-80^{\circ} \mathrm{C}$ pending biochemical analysis.

\section{Homogenization of brain tissue}

The brain extraction procedure was performed as described by Öhrfelt et al. with minor modifications [39]. Briefly, $100 \pm 10 \mathrm{mg}$ of brain tissue was homogenized on ice in $1 \mathrm{~mL}$ Tris- hydrochloride buffer $(10 \mathrm{mM}$ Tris- $\mathrm{HCl}$, $\mathrm{pH}$ 6.8) containing complete protease inhibitor (Roche Diagnostics $\mathrm{GmBH}$ ). Centrifugation of the homogenate was performed at $31,000 \mathrm{~g}$ for $1 \mathrm{~h}$ at $+4^{\circ} \mathrm{C}$ and the supernatant was collected (Tris fraction, i.e., soluble fraction). One milliliter of Tris-buffer containing 0.5\% Triton X-100 (Union Carbide Corporation) with complete protease inhibitor was added, and the pellet was homogenized on ice and sonicated using a micro-probe sonicator. The centrifugation step was repeated and the supernatant was collected $(0.5 \%$ Triton fraction, i.e., membrane-bound fraction). The same procedure was repeated by addition of Tris-buffer containing $2 \%$ Triton (2\% Triton fraction) and complete protease inhibitor, and again by addition of Tris-buffer containing $0.5 \%$ sodium dodecyl sulphate and complete protease inhibitor for a final centrifugation at $+12^{\circ} \mathrm{C}$ (SDS fraction, i.e., membrane-raft associated fraction). All supernatants were aliquoted and stored at $-80^{\circ} \mathrm{C}$ pending analysis. For protein quantitation, Protein DC assay (Bio-Rad Laboratories) reagent was used. This reagent contains a reducing agent and is detergent compatible.

\section{Analysis of CSF biomarkers}

The CSF analyses on A $\beta 1-42$, T-tau and P-tau ${ }_{181}$ levels were performed using commercially available assays from 


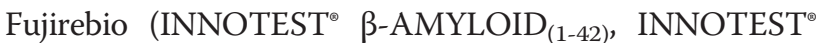
hTAU Ag and INNOTEST ${ }^{\circ}$ PHOSPHO-TAU(181P).

\section{Antibodies and recombinant protein of SNAP-25}

The following antibodies were used: mouse monoclonal antibody SP12 recognizing SNAP-25 [40,41] and mouse monoclonal antibody SMI81 (Covance) against SNAP-25 [25]. The SMI81 antibody recognizes an epitope containing the N-terminally acetylated first 11 amino acids of brain SNAP-25 [25]. Recombinant standard protein of SNAP-25 was purchased from Origene.

\section{Immunoprecipitation}

The immunoprecipitation method for brain tissue extracts and CSF samples was performed according to Öhrfelt et al. with minor modifications [39]. Briefly, an aliquot $(1 \mu \mathrm{g})$ of the mouse monoclonal antibody SP12 $(1 \mathrm{~g} / \mathrm{L})$ or the mouse monoclonal antibody SMI81 $(1 \mathrm{~g} / \mathrm{L})$ or IgG from murine serum (1 g/L, Sigma-Aldrich) (a negative control), was separately added to $100 \mu \mathrm{L}$ magnetic Dynabeads M-280 Sheep anti-mouse IgG (Invitrogen Corporation) and incubated $1 \mathrm{~h}$ on a rocking platform at room temperature. The beads were washed three times with $1 \mathrm{~mL}$ of PBS (10 mM Na-phosphate, $0.15 \mathrm{M} \mathrm{NaCl}, \mathrm{pH}$ 7.4). The antibodies were cross-linked using $20 \mathrm{mM}$ dimethyl pimelimidate dihydrochloride (Sigma-Aldrich) and 0.2 M triethanolamine ( $\mathrm{pH} 8.2$ Sigma-Aldrich) according to the manufacturer's product description. The cross-linked beads were washed two times in PBS and were blocked with RotiBlock (Carl Roth) for $1 \mathrm{~h}$ on a rocking platform at room temperature. Each brain tissue extract (soluble, membranebound, and membrane-raft associated proteins) (26 $\mu \mathrm{g}$ of total protein) and CSF samples (German cohort, $890 \mu \mathrm{L}$; Swedish cohort I, $700 \mu \mathrm{L}$; Swedish cohort II, $600 \mu \mathrm{L}$ ) were adjusted with $20 \%$ Triton and PBS to a final concentration of $0.2 \%$ Triton and a final volume of $1 \mathrm{~mL}$ ). Samples and magnetic beads were incubated overnight on a rocking platform at $+4^{\circ} \mathrm{C}$. The magnetic beads/sample solution was transferred to the KingFisher magnetic particle processor (Thermo Fisher Scientific), tube 1. The following three wash steps (tubes 2-4) were conducted for $10 \mathrm{~s}$ in $1 \mathrm{~mL}$ of each washing buffer: (tube 2) $0.025 \%$ Tween 20 in PBS, (tube 3) PBS and (tube 4) $50 \mathrm{mM}$ ammonium hydrogen carbonate $\left(\mathrm{NH}_{4} \mathrm{HCO}_{3}, \mathrm{pH}\right.$ 8.0). SNAP-25 was then eluted from the beads by adding $100 \mu \mathrm{L} 0.5 \%$ formic acid (FA) (tube 5) for $4 \mathrm{~min}$. The eluted fractions were transferred to $0.5 \mathrm{~mL}$ Protein LoBind Tube (Eppendorf AG) and dried in a vacuum centrifuge.

\section{Protein digestion and addition of heavy-isotope labeled peptide standards Brain tissue}

The dried immunoprecipitated brain tissue samples were dissolved in $10 \mu \mathrm{L}$ 0.1\% RapiGest SF Surfactant (Waters) in $50 \mathrm{mM} \mathrm{NH}_{4} \mathrm{HCO}_{3} 1 \mathrm{~h}$ in room temperature. Disulfide bonds were reduced by addition of $10 \mu \mathrm{L} 10 \mathrm{mM}$ dithiothreitol (Sigma-Aldrich) in $50 \mathrm{mM} \mathrm{NH} \mathrm{NCO}_{3}$ and incubation for $3 \mathrm{~min}$ at $+90^{\circ} \mathrm{C}$. After cooling to room temperature, $5 \mu \mathrm{L} 10 \mathrm{mM}$ iodoacetamide (Sigma-Aldrich) in $50 \mathrm{mM} \mathrm{NH} \mathrm{HCO}_{3}$ was added, and the samples were incubated in the dark at room temperature for $30 \mathrm{~min}$. Digestion was carried out by adding $5 \mu \mathrm{L}$ trypsin solution (1 $\mu \mathrm{g}$ Sequencing Grade Modified Trypsin [Promega] dissolved in $0.01 \%$ aqueous $\mathrm{HCl}[0.1 \mathrm{~g} / \mathrm{L}]$ and diluted to $5 \mathrm{mg} / \mathrm{L}$ in $50 \mathrm{mM} \mathrm{NH}_{4} \mathrm{HCO}_{3}$ ) and incubating overnight at $+37^{\circ} \mathrm{C}$. To reduce the amount of RapiGest SF Surfactant in the samples $2 \mu \mathrm{L} 10 \%$ aqueous trifluoroacetic acid was added (resulting in $\mathrm{pH}<2$ ). The samples were incubated $45 \mathrm{~min}$ at $+37^{\circ} \mathrm{C}$ and then centrifuged $(16,900 \mathrm{~g}, 10 \mathrm{~min}$, $+4^{\circ} \mathrm{C}$ ). Twenty-five microliters of the resulting supernatant of each sample was carefully transferred to $0.5 \mathrm{~mL}$ Protein LoBind Tubes (Eppendorf AG). A C-terminally isotopically labeled peptide, containing U-13C6, U-15 N4-arginine $[R]$ (aa 18-30, ADQLADESLEST[R]) was supplied by Sigma-Aldrich at over $95 \%$ peptide purity as determined by reversed phase HPLC. The peptide was dissolved and diluted in $0.1 \%$ aqueous $\mathrm{FA}$ to a final concentration of $5 \mathrm{fmol} / \mu \mathrm{L}$. A $25 \mu \mathrm{L}$ aliquot of the reference peptide was added to each immunoprecipitated brain homogenate sample after digestion and centrifugation.

\section{CSF}

The dried immunoprecipitated CSF samples were dissolved in $5 \mu \mathrm{L}$ of a mixture of five isotopically labeled peptides, containing U-13C6, U-15 N4-arginine [R], U-13C6, U-15 N1-leucine [L] or U-13C6, U-15 N2-lysine [K] and common for the N-terminal part of both SNAP-25A and SNAP-25B. The peptide standard supplied by Sigma Aldrich (aa 18-30, see above) was mixed with four HeavyPeptide FasTrack 1 standards (Thermo Fisher Scientific) ( $\sim .5 \mathrm{mg}$ dissolved in $1 \mathrm{~mL}$ MilliQ water) (aa Ac2-16, AEDADMRNE[L]EEMQR; aa 17-31, RADQ[L]ADESLE STRR; aa 18-31 ADQLADESLEST[R][R]; and aa 32-40 MLQLVEES[K]) and diluted in $50 \mathrm{mM} \mathrm{NH} \mathrm{NHCO}_{3}$ to a final concentration of $\sim 100 \mathrm{fmol} / \mu \mathrm{L}$ (aa 18-30) and $\sim 3 \mathrm{ng} / \mu \mathrm{L}$ (FasTrack 1 standards). Reduction of disulfide bonds ( $5 \mu \mathrm{L} 10 \mathrm{mM}$ dithiothreitol), alkylation $(5 \mu \mathrm{L}$ $10 \mathrm{mM}$ iodoacetamide), and digestion $(5 \mu \mathrm{L} 5 \mathrm{mg} / \mathrm{L}$ trypsin) were performed as described above. To stop the enzymatic activity $4 \mu \mathrm{L} 10 \%$ aqueous FA was added. The samples were centrifuged $\left(16,900 \mathrm{~g}, 10 \mathrm{~min},+4^{\circ} \mathrm{C}\right)$ and $20 \mu \mathrm{L}$ of each sample was transferred to LC-vials (SUN-SRi).

\section{SRM-MS analysis of immunoprecipitated SNAP-25 from brain tissue}

Aliquots $(25 \mu \mathrm{L})$ of the 1:1 mixtures of stable isotopelabeled standard peptide and immunoprecipitated SNAP-25 
were transferred to LC-vials (SUN-SRi) and analyzed by SRM-MS using an Accela 1250 pump (Thermo Fischer Scientific) coupled to a triple quadrupole mass spectrometer (TSQ Vantage, Thermo Fischer Scientific) with an IonMax source and HESI-II electrospray probe (Thermo Fischer Scientific). Mobile phases were $0.1 \%$ aqueous FA $(\mathrm{v} / \mathrm{v})(\mathrm{A})$ and $0.1 \% \mathrm{FA}$ in $84 \% \mathrm{ACN}$ in water (v/v) (B). Samples $(20 \mu \mathrm{L})$ were loaded directly onto a Hypersil Gold-C18 column, (length $50 \mathrm{~mm}$, inner diameter $2.1 \mathrm{~mm}$, particle size $5 \mu \mathrm{m}$ [Thermo Fischer Scientific]) with $0.1 \%$ aqueous FA at $100 \mu \mathrm{L} / \mathrm{min}$. After $2 \mathrm{~min}$ of loading, the peptides were eluted off the column using the following linear gradient steps: $0 \mathrm{~min} 0 \% \mathrm{~B} ; 4 \mathrm{~min}$ 17\%B; 12 min 23\%B; 15 min 100\%B. The global MS parameters were: positive ion mode; spray voltage $3.5 \mathrm{kV}$; vaporizer temperature $+350^{\circ} \mathrm{C}$; sheath gas pressure 40 psi; auxiliary gas pressure 25 arbitrary units; capillary temperature $+350^{\circ} \mathrm{C}$; collision gas pressure 1.9 mTorr. Pinpoint software version 1.3.0 (Thermo Fischer Scientific) was used for method optimization and data processing.

\section{LC-MS/MS analysis of immunoprecipitated SNAP-25 from brain tissue}

Aliquots $(25 \mu \mathrm{L})$ of the 1:1 mixtures of stable isotope labeled standard peptide and immunoprecipitated SNAP-25 protein were transferred to LC-vials (Waters) and analyzed by LC-MS/MS. The LC-MS/MS spectra were acquired with a electrospray-linear quadrupole ion trap-Fourier transform ion cyclotron resonance (ESI-LQIT-FTICR) mass spectrometer equipped with a 7 T magnet (LTQ FT Ultra, Thermo Fischer Scientific) coupled to a multi-dimensional nanoflow chromatography system (Ettan MDLC, GE Healthcare). A Zorbax $300 \mathrm{SB}-\mathrm{C} 18$ trap column (length $5 \mathrm{~mm}$, inner diameter $0.3 \mathrm{~mm}$, particle size $5 \mu \mathrm{m}$ [Agilent Technologies]) was used for on-line desalting and a reversed phase Zorbax $300 \mathrm{SBC} 18$ column (length $150 \mathrm{~mm}$, inner diameter $0.075 \mathrm{~mm}$, particle size $3.5 \mu \mathrm{m}$ [Agilent Technologies]) was used for high-resolution separation. Mobile phases were $0.1 \%$ aqueous FA (v/v) (A) and $0.1 \%$ FA in $84 \%$ $\mathrm{ACN}$ in water $(\mathrm{v} / \mathrm{v})(\mathrm{B})$. The separation was performed at a flow rate of approximately $250 \mathrm{~nL} / \mathrm{min}$ by applying a linear gradient of $0-60 \%$ B for $50 \mathrm{~min}$. The LTQ FT Ultra was set to acquire positive ions and operated in the data-dependent mode, where a scan cycle consisted of one full scan mass spectrum (m/z 350-1500) acquired in the FTICR mode (resolution 25,000), followed by tandem mass spectrometry (MS/MS) scans acquired in LQIT mode using collision-induced dissociation (CID) and wideband activation. Dynamic exclusion was enabled with repeat count 2 and exclusion duration $120 \mathrm{~s}$. Isolation width was $3 \mathrm{~m} / \mathrm{z}$ units, and the normalized collision energy to 35 . Each scan consisted of three microscans.

\section{Database search and bioinformatic analysis}

Database searches were submitted to the in-house database server by using Mascot Deamon 2.3.0 (Matrix Science). Database search parameters were; database (UniProtKB Human 131030), taxonomy (Homo sapiens), enzyme (trypsin), variable modifications (acetyl [N-term], oxidation [M], Label:13C(6)15 N(2) [K], and Label:13C(6)15 N (4) $[R]$ ), fixed modifications (Carbamidomethyl $[C]$ ), mass values (monoisotopic), peptide mass tolerance (5 ppm), fragment mass tolerance $(0.5 \mathrm{Da})$, and max missed cleavages (2).

\section{Quantification of high mass accuracy precursor ions}

Peak detection and integration were performed using DeCyder 2.0 (GE Healthcare) using processing parameters as described in [42]. In-house developed software (Sequence and PeakExtractor) was used for in silico digestion and automatic peak mass matching.

\section{Top-down-LC-MS/MS analysis of intact SNAP-25 fragments from brain tissue}

Soluble SNAP-25 forms immunoprecipitated with SMI81 from three different brain homogenate fractions were pooled in a $0.5 \mathrm{~mL}$ Protein LoBind Tube (Eppendorf AG) and dried in a vacuum centrifuge. The sample was dissolved in $25 \mu \mathrm{L}$ of $0.1 \%$ aqueous FA for $1 \mathrm{~h}$ and then centrifuged $\left(16,900 \mathrm{~g}, 10 \mathrm{~min},+4^{\circ} \mathrm{C}\right)$. The LC-MS/MS spectra were acquired with the Ettan MDLC/LTQ FT Ultra system using the same LC settings and columns as for the digested samples (see above). The LTQ FT Ultra was operated in data-dependent mode with a scan cycle consisting of one full scan mass spectrum acquired in FTICR mode (m/z 500-1,200, resolution 50,000), and one MS/MS scan in FTICR mode (resolution 50,000) using CID. Each scan consisted of three microscans. Isolation width was $7 \mathrm{~m} / \mathrm{z}$ units, and the normalized collision energy 35 . To ensure MS/MS acquisition for the peptides of interest, an inclusion mass list was utilized. Peak picking and charge deconvolution of acquired spectra were performed using Mascot Distiller (Matrix Science) with processing parameters as described in [42]. Database search parameters were; database (UniProtKB_Human 120809), taxonomy (Homo sapiens), enzyme (none), variable modifications (oxidation $[\mathrm{M}]$ ), fixed modifications (acetyl [N-term], carbamidomethyl $[\mathrm{C}]$ ), mass values (monoisotopic), peptide mass tolerance $(10 \mathrm{ppm})$, fragment mass tolerance $(50 \mathrm{mmu})$, and instrument type (FTICR CID Distiller, meaning only singly charged fragment ions were considered in the database search).

\section{HR-SIM-MS analysis of immunoprecipitated SNAP-25 from CSF}

High-resolution selected ion monitoring (HR-SIM) analyses were performed on a quadrupole-orbitrap mass 
spectrometer Q Exactive (Thermo Fisher Scientific) coupled to an Ultimate 3000 chromatography system (Thermo Fisher Scientific). The samples $(15 \mu \mathrm{L})$ were loaded directly onto a Hypersil Gold-C18 column, (see SRM-MS analysis) with $0.1 \%$ aqueous $\mathrm{FA}$ at $100 \mu \mathrm{L} / \mathrm{min}$. Mobile phases were the same as in the SRM-MS analysis. After 2 min of loading, the peptides were eluted off the column using the following linear gradient steps: 0 min $0 \% \mathrm{~B}$; 4 min 13\%B; 30 min 17\%B; 50 min 26\%B; 52 min $90 \% \mathrm{~B}$. The IonMax ion source settings were: spray voltage, $+4100 \mathrm{~V}$; capillary temperature, $+320^{\circ} \mathrm{C}$; sheath gas pressure, 25 arbitrary units; auxiliary gas pressure, 10 arbitrary units; and heater temperature, $+300^{\circ} \mathrm{C}$. The instrument was set to acquire scheduled pairs of SIM scans and subsequent all ion fragmentation scans in profile mode allowing simultaneous detection of both the SNAP-25 peptide and the corresponding isotopically labeled peptide standard. The settings were common for both scans types and were as follows: resolution, 70,000; AGC target, 3e6; maximum injection time, $300 \mathrm{~ms}$. Data acquisition and analysis were performed with Xcalibar software version 2.2 SP1.48 (Thermo Fisher Scientific) and Pinpoint 1.3.0. SNAP-25 levels for the different tryptic peptides were compensated for the different CSF volumes and reported as the ratio between the peak areas of the endogenous peptide and the labeled peptide standard multiplied by 10,000 .

\section{Investigation of reproducibility}

Approximately 5 pmol each of two isotopically labeled Nterminal SNAP-25 peptides (aa Ac2-16, AEDADM[R] NELEEMQ[R] and aa Ac2-20 AEDADMRNE[L]EEMQR RADQ, FasTrack 1 [Thermo Fisher Scientific]) were added to $15 \mathrm{~mL}$ CSF. The spiked CSF pool was divided into $890 \mu \mathrm{L}$ aliquots, immunoprecipitated with SMI81 and digested as described above. HR-SIM-MS analysis of eight of the samples was performed on $8 \mu \mathrm{L}$ injections. The CV of the measured levels was less than $10 \%$ (Additional file 1: Table S3).

\section{Statistical analysis}

Because the distributions of most analytes were not normal (Shapiro-Wilk test, $\mathrm{P}<0.05$ ), non-parametric statistics were used for analysis. Data are given as median (inter-quartile range). Differences between more than two groups were assessed with Kruskal-Wallis test. Statistically significant results $(\mathrm{P}<0.05)$ were followed by MannWhitney U-tests to investigate group differences. Since there were no significant alterations of the levels of novel SNAP-25 biomarkers, A $\beta 1-42$, and P-tau 181 between the different cohorts, it was possible to perform the receiver operating characteristic (ROC) curve analysis and assess correlations in all patients with Alzheimer's disease and controls. ROC curves were performed on each subject group on the tryptic peptides of SNAP-25 in order to assess their diagnostic value. For each tryptic peptide of SNAP-25 the area under the curve and a 95\% confidence interval was calculated using GraphPad Prism 5. The correlation coefficients (rho) were calculated using the Spearman two-tailed correlation test. SPSS 20.0 was employed for most of the statistical analyses.

\section{Ethics}

The present study was approved by the Regional Ethics Committee at the medical faculty Mannheim, University of Heidelberg, Germany and Lund University, Gothenburg University, Sweden. All patients gave their informed consent for research, which was conducted in accordance with the Helsinki Declaration The ethical principles abided by Netherlands Brain Bank are found at the website: (www.hersenbank.nl).

\section{Additional file}

Additional file 1: Table S1. Clinical and demographic characteristics of the brain tissue material. Table S2. Soluble SNAP-25 forms identified by LC-MS/MS. Table S3. HR-SIM-MS peak area of the human CSF tryptic SNAP-25 peptide Ac-2-16, and the spiked in isotopically labeled peptides $A c-2-16[R]$ and Ac-2-16[L]. Figure S1. Heat map for the relative signal intensities of individual tryptic peptides from SNAP-25B. Figure S2.

Tandem mass spectra of three different soluble N-terminal fragments of SNAP-25 acquired in the FTICR mode.

\section{Abbreviations}

Aß1-42: Amyloidß1-42; CSF: Cerebrospinal fluid; CID: Collision-induced dissociation; FA: Formic acid; FTICR: Fourier transform ion cyclotron resonance; HR-SIM-MS: High resolution-selected ion monitoring-mass spectrometry; LQIT: Linear quadrupole ion trap; LC-MS/MS: Liquid chromatography-tandem mass spectrometry; MCl: Mild cognitive impairment; MMSE: Mini-mental state examination; ROC: Receiver operating characteristic; SRM-MS: Selected reaction monitoring-mass spectrometry; SNARE: Soluble N-ethylmaleimide sensitive fusion attachment protein receptor; SNAP-25: Synaptosomal-associated protein 25; P-tau 181 : Tau phosphorylated at threonine 181; T-tau: Total tau.

\section{Competing interests}

The authors declare that they have no competing interests.

\section{Authors' contributions}

$A B$ and $A O ̈$ designed and performed the research; $A B, G B, W G H, H Z, K B$, and $A O ̈$ analyzed the data and interpreted results; $A O ̈$ performed statistical analyses; LF, LH, LM, OH, and AW recruited subjects and analyzed clinical data and all authors wrote the paper. All authors read and approved the final manuscript.

\section{Acknowledgements}

We are grateful to Rita Persson for her technical assistance. This work was supported by grants from the Swedish Brain Power consortium, Swedish Alzheimer Foundation, Swedish Research Council, ALF, the Knut and Alice Wallenberg Foundation, Demensfonden, Eivind och Elsa K:son Sylvans stiftelse, the Wolfson Foundation, Märtha och Gustaf Ågrens stiftelse, Gun och Bertil Stohnes stiftelse, Stiftelsen Gamla Tjänarinnor, Magn. Bergvalls stiftelse, Svenska Läkaresällskapet, the Canadian Institutes of Health Research (MOP-14037 and CBG-101827), Åhlén-stiftelsen and BMBF BIOMARK-APD (DLR $01 E D 1203 \mathrm{~J})$ 


\section{Author details}

'Institute of Neuroscience and Physiology, Department of Psychiatry and Neurochemistry, Sahlgrenska Academy at the University of Gothenburg, S-431 80 Mölndal, Sweden. ${ }^{2}$ Department of Psychiatry, University of British Columbia, Vancouver, Canada. ${ }^{3}$ Department of Geriatric Psychiatry, Central Institute for Mental Health Mannheim, University of Heidelberg, Mannheim, Germany. ${ }^{4}$ Clinical Memory Research Unit, Department of Clinical Sciences, Lund University, Lund, Sweden. ${ }^{5}$ Memory Clinic, Skåne University Hospital, Skåne, Sweden. ${ }^{6}$ UCL Institute of Neurology, Queen Square, London WC1N 3BG, London, UK.

Received: 8 July 2014 Accepted: 2 October 2014

Published: 23 November 2014

\section{References}

1. Busche MA, Eichhoff G, Adelsberger H, Abramowski D, Wiederhold KH, Haass C, Staufenbiel M, Konnerth A, Garaschuk O: Clusters of hyperactive neurons near amyloid plaques in a mouse model of Alzheimer's disease. Science 2008, 321:1686-1689.

2. Shankar GM, Li S, Mehta TH, Garcia-Munoz A, Shepardson NE, Smith I, Brett FM, Farrell MA, Rowan MJ, Lemere CA, Regan CM, Walsh DM, Sabatini BL, Selkoe DJ: Amyloid-beta protein dimers isolated directly from Alzheimer's brains impair synaptic plasticity and memory. Nat Med 2008, 14:837-842.

3. Blennow K, Bogdanovic N, Alafuzoff I, Ekman R, Davidsson P: Synaptic pathology in Alzheimer's disease: relation to severity of dementia, but not to senile plaques, neurofibrillary tangles, or the ApoE4 allele. J Neural Transm 1996, 103:603-618.

4. DeKosky ST, Scheff SW: Synapse loss in frontal cortex biopsies in Alzheimer's disease: correlation with cognitive severity. Ann Neurol 1990 27:457-464.

5. Honer WG: Pathology of presynaptic proteins in Alzheimer's disease: more than simple loss of terminals. Neurobiol Aging 2003, 24:1047-1062.

6. Honer WG, Barr AM, Sawada K, Thornton AE, Morris MC, Leurgans SE, Schneider JA, Bennett DA: Cognitive reserve, presynaptic proteins and dementia in the elderly. Transl Psychiatry 2012, 2:e114.

7. Masliah E, Mallory M, Alford M, DeTeresa R, Hansen LA, McKeel DW Jr, Morris JC: Altered expression of synaptic proteins occurs early during progression of Alzheimer's disease. Neurology 2001, 56:127-129.

8. Minger SL, Honer WG, Esiri MM, McDonald B, Keene J, Nicoll JA, Carter J, Hope T, Francis PT: Synaptic pathology in prefrontal cortex is present only with severe dementia in Alzheimer disease. J Neuropathol Exp Neurol 2001, 60:929-936.

9. Mukaetova-Ladinska EB, Garcia-Siera F, Hurt J, Gertz HJ, Xuereb JH, Hills R, Brayne C, Huppert FA, Paykel ES, McGee M, Jakes R, Honer WG, Harrington CR, Wischik CM: Staging of cytoskeletal and beta-amyloid changes in human isocortex reveals biphasic synaptic protein response during progression of Alzheimer's disease. Am J Pathol 2000, 157:623-636.

10. Scheff SW, Price DA, Schmitt FA, DeKosky ST, Mufson EJ: Synaptic alterations in CA1 in mild Alzheimer disease and mild cognitive impairment. Neurology 2007, 68:1501-1508.

11. Scheff SW, Price DA, Schmitt FA, Mufson EJ: Hippocampal synaptic loss in early Alzheimer's disease and mild cognitive impairment. Neurobiol Aging 2006, 27:1372-1384.

12. Terry RD, Masliah E, Salmon DP, Butters N, DeTeresa R, Hill R, Hansen LA, Katzman R: Physical basis of cognitive alterations in Alzheimer's disease: synapse loss is the major correlate of cognitive impairment. Ann Neurol 1991, 30:572-580

13. Blennow $\mathrm{K}$, Zetterberg $\mathrm{H}$ : The application of cerebrospinal fluid biomarkers in early diagnosis of Alzheimer disease. Med Clin North Am 2013, 97:369-376.

14. Jahn R, Sudhof TC: Membrane fusion and exocytosis. Annu Rev Biochem 1999, 68:863-911.

15. Sudhof TC, Rothman JE: Membrane fusion: grappling with SNARE and SM proteins. Science 2009, 323:474-477.

16. Davidsson P, Jahn R, Bergquist J, Ekman R, Blennow K: Synaptotagmin, a synaptic vesicle protein, is present in human cerebrospinal fluid: a new biochemical marker for synaptic pathology in Alzheimer disease? $\mathrm{Mol}$ Chem Neuropathol 1996, 27:195-210.

17. Davidsson P, Puchades $M$, Blennow K: Identification of synaptic vesicle, pre- and postsynaptic proteins in human cerebrospinal fluid using liquid-phase isoelectric focusing. Electrophoresis 1999, 20:431-437.
18. Thompson PM, Kelley M, Yao J, Tsai G, van Kammen DP: Elevated cerebrospinal fluid SNAP-25 in schizophrenia. Biol Psychiatry 2003, 53:1132-1137

19. Thompson PM, Rosenberger C, Holt S, Perrone-Bizzozero NI: Measuring synaptosomal associated protein-25 kDa in human cerebral spinal fluid. J Psychiatr Res 1998, 32:297-300.

20. Thorsell A, Bjerke M, Gobom J, Brunhage E, Vanmechelen E, Andreasen N, Hansson O, Minthon L, Zetterberg H, Blennow K: Neurogranin in cerebrospinal fluid as a marker of synaptic degeneration in Alzheimer's disease. Brain Res 2010, 1362:13-22

21. Zougman A, Pilch B, Podtelejnikov A, Kiehntopf M, Schnabel C, Kumar C, Mann M: Integrated analysis of the cerebrospinal fluid peptidome and proteome. J Proteome Res 2008, 7:386-399.

22. Gallien S, Duriez E, Crone C, Kellmann M, Moehring T, Domon B: Targeted proteomic quantification on quadrupole-orbitrap mass spectrometer. Mol Cell Proteomics 2012, 11:1709-1723.

23. Greber S, Lubec G, Cairns N, Fountoulakis M: Decreased levels of synaptosomal associated protein 25 in the brain of patients with Down syndrome and Alzheimer's disease. Electrophoresis 1999, 20:928-934.

24. Shimohama S, Kamiya S, Taniguchi T, Akagawa K, Kimura J: Differential involvement of synaptic vesicle and presynaptic plasma membrane proteins in Alzheimer's disease. Biochem Biophys Res Commun 1997, 236:239-242.

25. Connell E, Darios F, Peak-Chew S, Soloviev M, Davletov B: N-terminal acetylation of the neuronal protein SNAP-25 is revealed by the SMI81 monoclonal antibody. Biochemistry 2009, 48:9582-9589.

26. Fasshauer D, Sutton RB, Brunger AT, Jahn R: Conserved structural features of the synaptic fusion complex: SNARE proteins reclassified as Q- and R-SNAREs. Proc Natl Acad Sci U S A 1998, 95:15781-15786.

27. Sutton RB, Fasshauer $D$, Jahn R, Brunger AT: Crystal structure of a SNARE complex involved in synaptic exocytosis at 2.4 A resolution. Nature 1998, 395:347-353.

28. Gosso MF, de Geus EJ, Polderman TJ, Boomsma DI, Heutink P, Posthuma D: Common variants underlying cognitive ability: further evidence for association between the SNAP-25 gene and cognition using a family-based study in two independent Dutch cohorts. Genes Brain Behav 2008, 7:355-364.

29. Guerini FR, Agliardi C, Sironi M, Arosio B, Calabrese E, Zanzottera M, Bolognesi E, Ricci C, Costa AS, Galimberti D, Griffanti L, Bianchi A, Savazzi F, Mari D, Scarpini E, Baglio F, Nemni R, Clerici M: Possible Association between SNAP-25 Single Nucleotide Polymorphisms and Alterations of Categorical Fluency and Functional MRI Parameters in Alzheimer's Disease. J Alzheimers Dis 2014, 42:1015-1028.

30. Blennow K, Hampel H, Weiner M, Zetterberg H: Cerebrospinal fluid and plasma biomarkers in Alzheimer disease. Nat Rev Neurol 2010, 6:131-144.

31. Blennow K, Zetterberg H, Minthon L, Lannfelt L, Strid S, Annas P, Basun H, Andreasen N: Longitudinal stability of CSF biomarkers in Alzheimer's disease. Neurosci Lett 2007, 419:18-22.

32. Salomone S, Caraci F, Leggio GM, Fedotova J, Drago F: New pharmacological strategies for treatment of Alzheimer's disease: focus on disease modifying drugs. Br J Clin Pharmacol 2012, 73:504-517.

33. Braak $H$, Braak E: Neuropathological stageing of Alzheimer-related changes. Acta Neuropathol 1991, 82:239-259.

34. McKhann G, Drachman D, Folstein M, Katzman R, Price D, Stadlan EM: Clinical diagnosis of Alzheimer's disease: report of the NINCDS-ADRDA Work Group under the auspices of Department of Health and Human Services Task Force on Alzheimer's Disease. Neurology 1984, 34:939-944

35. Albert MS: Changes in cognition. Neurobiol Aging 2011, 32 Suppl 1:S58-S63.

36. American Psychiatric Association: Diagnostic and Statistical Manual of Mental Disorders. 4th edition. Washington DC: American Psychiatric Association; 1994.

37. Nordlund A, Rolstad S, Hellstrom P, Sjogren M, Hansen S, Wallin A: The Goteborg MCl study: mild cognitive impairment is a heterogeneous condition. J Neurol Neurosurg Psychiatry 2005, 76:1485-1490.

38. World Health Organization: The ICD-10 Classification of Mental and Behavioural Disorders. Geneva; 1992

39. Öhrfelt A, Zetterberg H, Andersson K, Persson R, Secic D, Brinkmalm G, Wallin A, Mulugeta E, Francis PT, Vanmechelen E, Aarsland D, Ballard C, Blennow K, Westman-Brinkmalm A: Identification of Novel alpha-Synuclein Isoforms in Human Brain Tissue by using an Online NanoLC-ESI-FTICR-MS Method. Neurochem Res 2011, 36:2029-2042. 
40. Honer WG, Falkai P, Young C, Wang T, Xie J, Bonner J, Hu L, Boulianne GL, Luo Z, Trimble WS: Cingulate cortex synaptic terminal proteins and neural cell adhesion molecule in schizophrenia. Neuroscience 1997, 78:99-110.

41. Oyler GA, Higgins GA, Hart RA, Battenberg E, Billingsley M, Bloom FE, Wilson MC: The identification of a novel synaptosomal-associated protein, SNAP-25, differentially expressed by neuronal subpopulations. J Cell Biol 1989, 109:3039-3052.

42. Brinkmalm G, Portelius E, Ohrfelt A, Mattsson N, Persson R, Gustavsson MK, Vite CH, Gobom J, Mansson J-E, Nilsson J, Halim A, Larson G, Ruetschi U, Zetterberg H, Blennow K, Brinkmalm A: An online nano-LC-ESI-FTICR-MS method for comprehensive characterization of endogenous fragments from amyloid beta and amyloid precursor protein in human and cat cerebrospinal fluid. J Mass Spectrom 2012, 47:591-603.

doi:10.1186/1750-1326-9-53

Cite this article as: Brinkmalm et al: SNAP-25 is a promising novel cerebrospinal fluid biomarker for synapse degeneration in Alzheimer's disease. Molecular Neurodegeneration 2014 9:53.

\section{Submit your next manuscript to BioMed Central and take full advantage of:}

- Convenient online submission

- Thorough peer review

- No space constraints or color figure charges

- Immediate publication on acceptance

- Inclusion in PubMed, CAS, Scopus and Google Scholar

- Research which is freely available for redistribution 